\title{
Front Matter: Volume 7920
}

, "Front Matter: Volume 7920," Proc. SPIE 7920, Laser Applications in Microelectronic and Optoelectronic Manufacturing (LAMOM) XVI, 792001 (16 March 2011); doi: 10.1117/12.890189

SPIE. Event: SPIE LASE, 2011, San Francisco, California, United States 


\section{PROCEEDINGS OF SPIE}

\section{Laser Applications in Microelectronic and Optoelectronic Manufacturing (LAMOM) XVI}

Bo Gu

Guido Hennig

Xianfan XU

Hiroyuki Niino

Editors

24-27 January 2011

San Francisco, California, United States

Sponsored by

SPIE

Cosponsored by

IPG (Beijing) Fiber Laser Technology Co., Ltd. (China)

Published by

SPIE

Volume 7920

Proceedings of SPIE, 0277-786X, v. 7920 
The papers included in this volume were part of the technical conference cited on the cover and title page. Papers were selected and subject to review by the editors and conference program committee. Some conference presentations may not be available for publication. The papers published in these proceedings reflect the work and thoughts of the authors and are published herein as submitted. The publisher is not responsible for the validity of the information or for any outcomes resulting from reliance thereon.

Please use the following format to cite material from this book:

Author(s), "Title of Paper," in Laser Applications in Microelectronic and Optoelectronic Manufacturing (LAMOM) XVI, edited by Bo GU, Guido Hennig, Xianfan XU, Hiroyuki Niino, Proceedings of SPIE Vol. 7920 (SPIE, Bellingham, WA, 2011) Article CID Number.

ISSN 0277-786X

ISBN 9780819484574

Published by

SPIE

P.O. Box 10, Bellingham, Washington 98227-0010 USA

Telephone +1 3606763290 (Pacific Time) · Fax +1 3606471445

SPIE.org

Copyright (@ 2011, Society of Photo-Optical Instrumentation Engineers

Copying of material in this book for internal or personal use, or for the internal or personal use of specific clients, beyond the fair use provisions granted by the U.S. Copyright Law is authorized by SPIE subject to payment of copying fees. The Transactional Reporting Service base fee for this volume is $\$ 18.00$ per article (or portion thereof), which should be paid directly to the Copyright Clearance Center (CCC), 222 Rosewood Drive, Danvers, MA 01923. Payment may also be made electronically through CCC Online at copyright.com. Other copying for republication, resale, advertising or promotion, or any form of systematic or multiple reproduction of any material in this book is prohibited except with permission in writing from the publisher. The CCC fee code is 0277-786X/11/\$18.00.

Printed in the United States of America.

Publication of record for individual papers is online in the SPIE Digital Library.

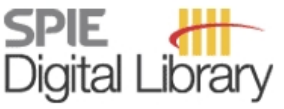

SPIEDigitalLibrary.org

Paper Numbering: Proceedings of SPIE follow an e-First publication model, with papers published first online and then in print and on CD-ROM. Papers are published as they are submitted and meet publication criteria. A unique, consistent, permanent citation identifier (CID) number is assigned to each article at the time of the first publication. Utilization of CIDs allows articles to be fully citable as soon they are published online, and connects the same identifier to all online, print, and electronic versions of the publication. SPIE uses a six-digit CID article numbering system in which:

- The first four digits correspond to the SPIE volume number.

- The last two digits indicate publication order within the volume using a Base 36 numbering system employing both numerals and letters. These two-number sets start with 00, 01, 02, 03, 04 , 05, 06, 07, 08, 09, OA, OB ... 0Z, followed by 10-1Z, 20-2Z, etc.

The CID number appears on each page of the manuscript. The complete citation is used on the first page, and an abbreviated version on subsequent pages. Numbers in the index correspond to the last two digits of the six-digit CID number. 


\section{Contents}

vii Conference Committee

ix Microfabrication by optical tweezers [792102]

R. Ghadiri, T. Weigel, C. Esen, A. Ostendorf, Ruhr-Univ. Bochum (Germany)

\section{FUNDAMENTAL ASPECTS OF LASER INTERACTION}

$792002 \quad$ Zinc ion and neutral emission from single crystal zinc oxide during 193-nm excimer laser exposure (Invited Paper) [7920-01]

E. Khan, S. C. Langford, Washington State Univ. (United States); L. A. Boatner, Oak Ridge National Lab. (United States); J. T. Dickinson, Washington State Univ. (United States)

792003 Absorption of femtosecond laser pulse in fused silica: experiments and modeling (Best Student Paper Award) [7920-02]

N. Varkentina, O. Utéza, N. Sanner, B. Chimier, M. Sentis, Lab. LP3, CNRS, Univ. de la Méditerranée (France); T. Itina, Lab. Hubert Curien, CNRS, Univ. Jean Monnet (France)

$792005 \quad$ Effects of the source, surface, and sensor couplings and colorimetric of laser speckle pattern on the performance of optical imaging system [7920-04]

M. Darwiesh, A. F. El-Sherif, Military Technical College (Egypt); H. El-Ghandour, Ain Shams Univ. (Egypt); H. A. Aly, A. M. Mokhtar, Military Technical College (Egypt)

\section{LASER MICROSCALE MATERIALS PROCESSING}

792006 Glass welding technology using ultra short laser pulses (Invited Paper) [7920-05] S. Roth, BLZ Bayerisches Laserzentrum GmbH (Germany) and Friedrich-Alexander Univ. Erlangen-Nürnberg (Germany); K. Cvecek, BLZ Bayerisches Laserzentrum GmbH (Germany); I. Miyamoto, Osaka Univ. (Japan) and Friedrich-Alexander Univ. Erlangen-Nürnberg (Germany); M. Schmidt, BLZ Bayerisches Laserzentrum GmbH (Germany) and Lehrstuhl für Photonische Technologien (Germany) and Friedrich-Alexander Univ. Erlangen-Nürnberg (Germany)

$792007 \quad$ Reliable laser micro-welding of copper [7920-06] C. Rüttimann, U. Dürr, LASAG AG (Switzerland); A. Moalem, M. Priehs, Laser Zentrum Hannover e.V. (Germany)

792008 Advanced micro-machining applications for low nanosecond low $\mathbf{M}^{2}$ fiber lasers [7920-07] T. Hoult, J. C. Kabahit, IPG Photonics Corp. (United States)

792009 Processing of metals with ps-laser pulses in the range between 10ps and 100ps [7920-08] M. Schmid, B. Neuenschwander, V. Romano, B. Jaeggi, U. W. Hunziker, Berner Fachhochschule Technik und Informatik (Switzerland) 
7920 OB Generation of superfine structure smaller than $10 \mathrm{~nm}$ by interfering femtosecond laser processing (Invited Paper) [7920-10]

Y. Nakata, K. Momoo, T. Hiromoto, N. Miyanaga, Osaka Univ. (Japan)

7920 OC Surface morphology of $\mathrm{SiO}_{2}$ coated InP/InGaAs/InGaAsP microstructures following irradiation with the ArF and KrF excimer lasers [7920-11]

N. Liu, K. Moumanis, J. J. Dubowski, Univ. de Sherbrooke (Canada)

\section{LASER MODIFICATION OF MATERIALS}

7920 ol Sintering of solution-based nano-particles by a UV laser pulse train [7920-17]

J. Zhang, M. Li, K. Morimoto, Panasonic Corp. of North America (United States)

THIN FILM AND WAFER PROCESSING: JOINT SESSION WITH CONFERENCE 7925

7920 OM Investigation on solid state $\mathrm{Nd}^{3+}$ :YAG line beam laser annealing and texturing of amorphous silicon thin films [7920-21]

N. J. Vasa, A. I. Palani, M. Singaperumal, Indian Institute of Technology Madras (India)

FEMTOSECOND LASER NANOPROCESSING: JOINT SESSION WITH CONFERENCE 7925

7920 OP Nanostructure formation on silicon surfaces by high repetition-rate sub- 15 femtosecond near-infrared laser pulses [7920-26]

M. Straub, K. König, Univ. des Saarlandes (Germany)

$79200 Q \quad$ Optimization methods of hologram for holographic femtosecond laser processing [7920-28]

Y. Hayasaki, S. Hasegawa, Utsunomiya Univ. (Japan)

\section{LASER PROCESSING OF TRANSPARENT MATERIALS}

7920 ОT Novel applications of sub-surface laser machining [7920-31]

B. R. Campbell, L. A. Forster, D. M. Bernot, The Pennsylvania State Univ. Electro-Optics Ctr. (United States)

$7920 \mathrm{OU} \quad \mathrm{CO}_{2}$ laser scribe of chemically strengthened glass with high surface compressive stress [7920-32]

X. Li, B. R. Vaddi, Corning Inc. (United States)

PROCESSING WITH NOVEL LASER SYSTEMS AND OPTICS

7920 OW Laser surface structuring with long depth of focus (Invited Paper) [7920-34]

A. Michalowski, C. Freitag, R. Weber, T. Graf, Univ. Stuttgart (Germany)

7920 0X A study on 8 beam laser diode to adopt laser scanning unit [7920-35]

D.-G. Song, J.-K. Park, J.-H. Park, A.-S. Choi, T.-K. Kim, QSI Co., Ltd. (Korea, Republic of) 
7920 OY Advantages offered by high average power picosecond lasers [7920-36]

C. Moorhouse, Coherent Scotland Ltd. (United Kingdom)

$79200 Z \quad$ Novel 1.2kW UV laser system for micro fabrication and annealing [7920-37]

L. Herbst, R. Paetzel, K. Schmidt, Coherent GmbH (Germany)

792010 Three-dimensional structuring inside transparent materials by a phase modulated fs laser beam with a LCOS-SLM (Invited Paper) [7920-38]

M. Sakakura, K. Miura, Kyoto Univ. (Japan); T. Sawano, New Glass Forum (Japan);

Y. Shimotsuma, K. Hirao, Kyoto Univ. (Japan)

PHOTOVOLTAICS/ENERGY DEVICES: JOINT SESSION WITH CONFERENCE 7921

792011 Innovative laser based solar cell scribing (Invited Paper) [7920-39]

B. Frei, S. Schneeberger, R. Witte, Solneva SA (Switzerland)

POSTER SESSION

792013 Generation of complicated or duplicated structure by interfering femtosecond laser processing of metallic thin film (Best Student Poster Award) [7920-41]

T. Hiromoto, K. Momoo, Y. Nakata, N. Miyanaga, Osaka Univ. (Japan)

792015 Multiphoton lithography and ITO structuring by high repetition-rate sub-15 femtosecond laser pulses [7920-43]

M. Afshar, S. Saremi, H. Völlm, D. Feili, H. Seidel, M. Straub, H. Zhang, K. König, Saarland Univ. (Germany)

792018 Evaluation of a refractive index profile for a modification induced by focused femtosecond laser irradiation in the optical glasses [7920-46]

T. Hashimoto, S. Tanaka, New Glass Forum (Japan)

792019 Laser cutting of carbon fiber reinforced plastics (CFRP) by UV pulsed laser ablation [7920-47] H. Niino, National Institute of Advanced Industrial Science and Technology (Japan) and Advanced Laser and Process Technology Research Association (Japan); R. Kurosaki, National Institute of Advanced Industrial Science and Technology (Japan)

7920 1 A $\quad \mathrm{F}_{2}$ laser formation of $\mathrm{SiO}_{2}$ protective layer onto polycarbonate for lightweight vehicle window [7920-48]

S. Sonobe, National Defense Academy (Japan); Y. Nojima, National Defense Academy (Japan) and Renias Co., Ltd. (Japan); M. Okoshi, National Defense Academy (Japan);

H. Nojiri, Renias Co., Ltd. (Japan); N. Inove, National Defense Academy (Japan)

7920 1B Patterning of aluminum thin films by $157 \mathrm{~nm} \mathrm{~F}_{2}$ laser [7920-49]

K. Iwai, M. Okoshi, National Defense Academy (Japan); H. Nojiri, Renias Co., Ltd. (Japan);

N. Inove, National Defense Academy (Japan)

Author Index 
Downloaded From: https://www.spiedigitallibrary.org/conference-proceedings-of-spie on 26 Apr 2023

Terms of Use: https://www.spiedigitallibrary.org/terms-of-use 


\title{
Conference Committee
}

\author{
Symposium Chairs
}

Friedhelm Dorsch, TRUMPF GmbH \& Company KG (Germany)

Alberto Piqué, Naval Research Laboratory (United States)

Symposium Cochairs

Donald J. Harter, IMRA America, Inc. (United States)

Peter R. Herman, University of Toronto (Canada)

Program Track Chairs

Henry Helvajian, The Aerospace Corporation (United States)

James S. Horwitz, U.S. Department of Energy (United States)

\section{Conference Chairs}

Bo Gu, IPG Photonics Corpration (United States)

Guido Hennig, Daetwyler Graphics AG (Switzerland)

Xianfan Xu, Purdue University (United States)

Hiroyuki Niino, National Institute of Advanced Industrial Science and Technology (Japan)

\section{Program Committee}

Craig B. Arnold, Princeton University (United States)

Ian W. Boyd, Monash University (Australia)

Paul V. Braun, University of Illinois at Urbana-Champaign (United States)

Douglas B. Chrisey, Rensselaer Polytechnic Institute (United States)

Santiago Costantino, Université de Montréal (Canada)

J. Thomas Dickinson, Washington State University (United States)

Jan J. Dubowski, Université de Sherbrooke (Canada)

Richard F. Haglund, Jr., Vanderbilt University (United States)

Yoshio Hayasaki, Utsunomiya University (Japan)

Andrew S. Holmes, Imperial College London (United Kingdom)

Jyotirmoy Mazumder, University of Michigan (United States)

Eric D. Mazur, Harvard University (United States)

Michel Meunier, Ecole Polytechnique de Montréal (Canada)

Beat Nevenschwander, Berner Fachhochschule (Switzerland)

Henry Peng, GE China Technology Center (China)

Alberto Piqué, U.S. Naval Research Laboratory (United States)

Koji Sugioka, RIKEN (Japan)

Vassilia Zorba, Lawrence Berkeley National Laboratory (United States) 
Session Chairs

1 Fundamental Aspects of Laser Interaction

Bo Gu, IPG Photonics Corporation (United States)

Guido Hennig, Daetwyler Graphics AG (Switzerland)

2 Laser Microscale Materials Processing

J. Thomas Dickinson, Washington State University (United States)

Yongfeng Lu, University of Nebraska-Lincoln (United States)

3 Surface Microstructuring and Cleaning

Guido Hennig, Daetwyler Graphics AG (Switzerland)

Bo Gu, IPG Photonics Corporation (United States)

$4 \quad$ Laser Modification of Materials

Hiroyuki Niino, National Institute of Advanced Industrial Science and Technology (Japan)

Yoshiki Nakata, Osaka University (Japan)

$5 \quad$ Ultrashort Pulse Micromachining: Joint Session with Conference 7925

Craig B. Arnold, Princeton University (United States)

6 Thin Film and Wafer Processing: Joint Session with Conference 7925

Jens Koenig, Robert Bosch GmbH (Germany)

7 Online Monitoring: Joint Session with Conference 7925

Andreas Michalowski, Universität Stuttgart (Germany)

8 Femtosecond Laser Nanoprocessing: Joint Session with Conference

7925

Beat Neuenschwander, Berner Fachhochschule Technik und Informatik (Switzerland)

9 Laser Processing of Transparent Materials

Xianfan Xu, Purdue University (United States)

Andreas Michalowski, Universität Stuttgart (Germany)

10 Processing with Novel Laser Systems and Optics

Razvan Stoian, Laboratoroire Hubert Curien, CNRS, Université de Lyon, Université Jean Monnet Saint-Etienne (France)

Hiroyuki Niino, National Institute of Advanced Industrial Science and Technology (Japan)

11 Photovoltaics/Energy Devices: Joint Session with Conference 7921

Yongfeng Lu, University of Nebraska-Lincoln (United States) 